ISSN 1808-3765

\title{
PERDA DE CARGA EM MANGUEIRAS GOTEJADORAS NOVAS E USADAS
}

\author{
Jayme Laperuta Neto, Raimundo Leite Cruz, Jayme Laperuta Filho, Thalita Aparecida \\ Pletsch \\ Departamento de Engenharia Rural, Faculdade de Ciências Agronômicas, Universidade Estadual Paulista, \\ Botucatu, SP, neto neo@fca.unesp.br
}

\section{RESUMO}

O presente trabalho teve como objetivo determinar a importância da perda de carga localizada de um emissor tipo gotejador no dimensionamento da linha lateral, bem como comparar os resultados das equações que calculam a perda de carga localizada, além de verificar a influência de precipitados férricos, na perda de carga de uma mangueira gotejadora fabricada no Brasil. Como resultado obteve-se que a perda de carga representou $23 \%$ da perda de carga total, as equações apresentaram resultados significativos e a perda de carga aumentou em média $20 \%$ após o uso do sistema com água rica a em ferro.

Palavras-chave: Irrigação Localizada, Perda de carga localizada.

\section{LAPERUTA NETO, J.; CRUZ, R. L., LAPERUTA FILHO, J., PLETSCH, T. A. HEAD LOSS IN USED AND NEW HOSE DRIP LINES}

\section{ABSTRACT}

This paper aims to determine the importance of emitter's local head loss in the hydraulic design of hose drip lines, as well as comparing the result of equations that calculate the local head loss; besides verifying the influence of iron precipitated at the hose head loss made in Brazil. The result found that localized head loss represents $23 \%$ of the total head loss; the equations showed significant results and the total head loss increased in an average of $20 \%$ after the irrigation system was used with water rich in iron.

KEY WORDS: Drip irrigation, local head loss.

\section{INTRODUÇÃO}

O contínuo crescimento da população mundial vem exigindo uma agricultura competitiva e tecnificada para aumentar a produção de alimentos.

Com o desenvolvimento da tecnologia do plástico e com a crescente necessidade de se economizar água, o sistema de irrigação por gotejamento vem se destacando por apresentar características como o uso racional da água, a qual é depositada na região radicular da cultura 
com um alto grau de uniformidade de aplicação da água, levando a cultura desenvolver-se uniformemente por toda a área.

Para que o sistema de irrigação funcione em sua plenitude, na elaboração do projeto é calculada a perda de carga total, composta pela perda de carga distribuída ao longo da tubulação e as perdas de carga localizadas, no intuito de se obter os diâmetros das tubulações e as características do conjunto moto-bomba.

Segundo Alves \& Porto (2002), no dimensionamento das instalações de irrigação por gotejamento são determinadas as perdas de carga distribuídas ao longo dos tubos, as perdas de carga localizada devido às singularidades nas linhas primárias e de derivação e as perdas localizadas devido aos gotejadores nas linhas laterais.

Segundo Juana et al. (2002), a perda de carga distribuída ao longo das laterais de sistemas de irrigação por gotejamento afeta a pressão disponível no bocal do emissor, conseqüentemente, a distribuição é significativamente afetada quando se usa gotejadores convencionais (não-autocompensantes). Por esse motivo o dimensionamento da linha lateral é função da variação de pressão e da variação da vazão entre o primeiro e o ultimo gotejador na linha lateral, variação esta que não deve exceder $10 \%$ da vazão média dos gotejadores, ao longo da lateral (Bernardo et al., 2006), o que é equivalente a um coeficiente de uniformidade de $98 \%$ (Wu, 1997).

Existem vários métodos de se calcular a perda de carga distribuída, o mais bem aceito é o calculado através da equação de Darcy-Weisbach, também chamada de fórmula Universal. A Equação 1 apresenta a fórmula Universal reescrita por Bagarello et al. (1995):

$$
J=0,0235 \frac{\vartheta^{0,25} Q^{1,75}}{D^{4,75}}
$$

Onde: $\mathrm{J}$ - perda de carga por comprimento de tubo; $\vartheta$ - coeficiente de viscosidade cinemática; D - Diâmetro da tubulação $(\mathrm{m}) ; \mathrm{Q}$ - vazão $\left(\mathrm{m}^{3} \mathrm{~s}^{-1}\right)$.

A perda de carga localizada ao longo de uma linha lateral devido às singularidades produzidas pela inserção de emissores sobre a linha, na linha e integrados, pode ser calculada pela Equação 2:

$$
\lambda_{i}=\alpha \frac{V_{i}^{2}}{2 g}
$$

Onde: $\lambda_{\mathrm{i}}-$ Perda de

das linhas de fluxo, respectivamente antes e depois do emissor e pode ser calculado carga localizada (L); $\mathrm{V}_{\mathrm{i}}$-Velocidade do fluído $\left(\mathrm{L} \mathrm{T}^{-1}\right) ; \mathrm{g}$ - aceleração da gravidade $\left(\mathrm{L} \mathrm{T}^{-2}\right) ; \alpha$ - coeficiente de carga cinética ou resistência do perfil.

Segundo Provenzano \& Pumo (2005), $\alpha$ é um coeficiente que sintetiza a perda de carga localizada devido à contração e o alargamento pela Equação 3:

$$
\alpha=0,116\left[\left(\frac{D_{i}}{D_{g}}\right)^{13,87}-1\right]
$$

Com: $1,00<\mathrm{D}_{\mathrm{i}} / \mathrm{D}_{\mathrm{g}}<1,20$ 
Onde: $\mathrm{D}_{\mathrm{i}}$ - Diâmetro interno da seção da mangueira gotejadora sem o emissor; $\mathrm{D}_{\mathrm{g}}$ Diâmetro interno da seção da mangueira gotejadora com o emissor.

Outro fator que influencia no funcionamento do sistema de irrigação é o entupimento. O entupimento do emissor convencional causa redução da sua vazão, o que acarreta uma diminuição da uniformidade do sistema, trazendo prejuízo à cultura. Causa também alteração na carga hidráulica dentro da mangueira gotejadora podendo provocar um desequilíbrio hidráulico no sistema.

O objetivo deste trabalho é comparar a perda de carga localizada, devido à presença do emissor na linha, obtida em ensaio e a obtida através das equações, além de verificar o efeito sobre a perda de carga de uma linha lateral após o uso de água rica em ferro (Tabela 1), para uma mangueira gotejadora com emissores coextrusados do tipo pastilha, modelo Tiran da Netafim.

Tabela 1. Resultados da Análise da água utilizada nos ensaios.

\begin{tabular}{lcc}
\hline Parâmetros & Unidade & Valores \\
\hline $\mathrm{pH}$ & & 4,55 \\
Condutividade elétrica & $\mu . \mathrm{S}^{-1} \cdot \mathrm{cm}^{-1}$ & 21,10 \\
Nitrato & & \\
$\mathrm{NO}_{3}^{-}-\mathrm{N}$ & $\mathrm{mg}^{-1} .1$ & 0,3 \\
$\mathrm{NO}_{3}^{-}$ & $\mathrm{mg}^{-1} \cdot 1$ & 55 \\
$\mathrm{Cor}$ & $\mathrm{UHT}$ & 55 \\
$\mathrm{DQO}$ & $\mathrm{mg}^{-1} \cdot 1$ & 0 \\
Fe & $\mathrm{mg}^{-1} \cdot 1$ & 3,5 \\
Turbidez & $\mathrm{FAU}$ & 13 \\
\hline
\end{tabular}

\section{MATERIAL E MÉTODOS}

\subsection{Instalação, equipamentos e montagem}

Os ensaios foram realizados no laboratório de ensaios de equipamentos de irrigação do Departamento de Engenharia Rural da Faculdade de Ciências Agronômicas - UNESP Botucatu, São Paulo.

Para a realização dos ensaios utilizou-se bancada de ensaio (Figura 1),composta por um reservatório localizado no início do sistema, uma bomba elétrica que abastece e pressuriza o sistema com a água proveniente do reservatório, um filtro de disco de 200 mesh para garantir que a água fornecida ao sistema esteja livre de partículas sólidas que possam influenciar nos resultados, ventosa no início da linha de gotejadores ligada ao sistema, como garantia de que todo ar, que possa estar dentro do sistema, seja expelido, manômetro e registro para regulagem de pressão na entrada do sistema e uma mangueira de retorno que conduzia o restante do líquido que passava pela mangueira gotejadora de volta ao tanque de abastecimento. 
A linha lateral foi composta de mangueira gotejadora com emissores coextrusados do tipo pastilha, com espaçamento de $50 \mathrm{~cm}$, ligada ao sistema com comprimento de aproximadamente $9,2 \mathrm{~m}$, resultando num trecho com 18 emissores no total.

B - Bomba Elétrica

F - Filtro de Disco

$V$ - Ventosa

R - Regulador de Pressäo

M - Manômetro Bourdon

$\mathrm{Pi}$ - Ponto de Tomada de Pressáo

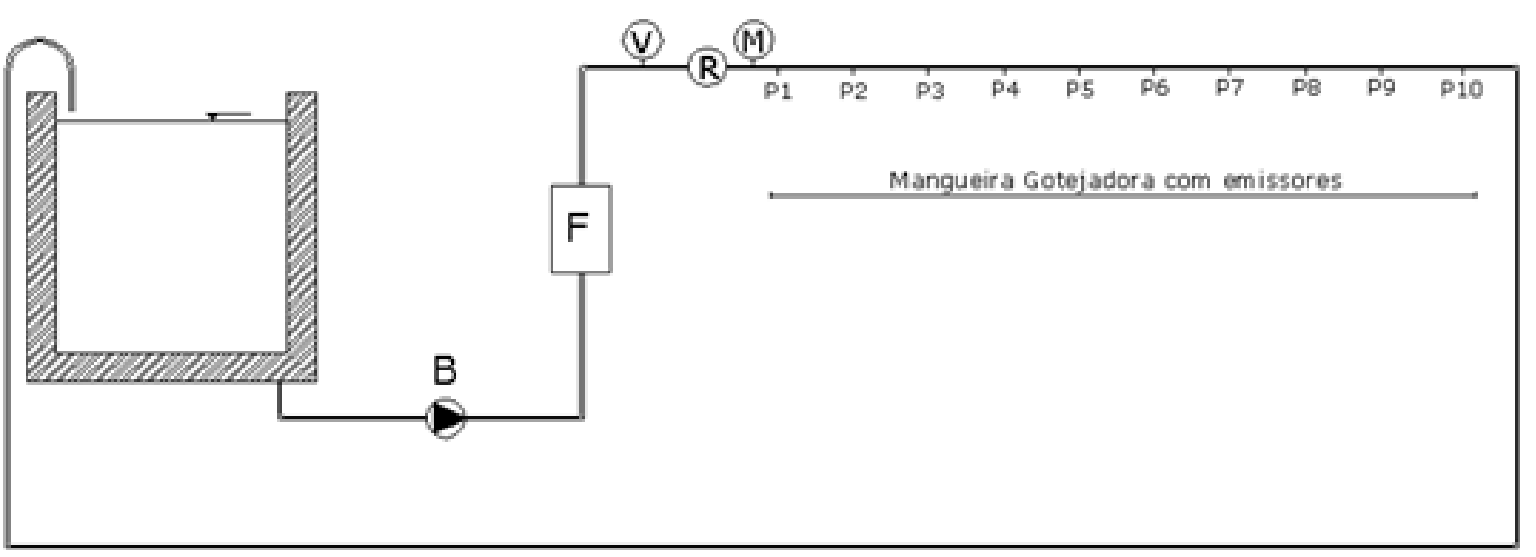

Figura 1. Bancada de ensaios.

Foram determinados dez pontos para se medir a pressão ao longo do comprimento da mangueira gotejadora, sendo que o primeiro e o último ficavam a $0,35 \mathrm{~m}$ do início e do fim da mangueira respectivamente. Os demais pontos ficavam espaçados a cada 1,0m. Em cada um desses pontos foram inseridas agulhas de soro pelas quais o tubo em $U$ de mercúrio (manômetro de mercúrio) era conectado ao sistema. Devido às reduzidas dimensões dessas agulhas foi desprezada a perda de carga causada pela sua inserção ao sistema.

Foi utilizada no experimento a pressão de $100 \mathrm{KPa}$, fornecida pelo fabricante como sendo a pressão de serviço do emissor, e uma vazão de aproximadamente $10501 . \mathrm{h}^{-1}$, que correspondeu a uma velocidade de aproximadamente $1,80 \mathrm{~m} \cdot \mathrm{s}^{-1}$. Durante o experimento foi observada a temperatura da água para o cálculo da viscosidade cinemática da água.

De posse dos valores da pressão em cada ponto obteve-se a perda de carga, trecho a trecho, subtraindo a pressão no primeiro ponto com a pressão no ponto analisado.

O procedimento foi repetido para três amostras da mangueira gotejadora, extraídas ao acaso, quando novas e após serem submetidas a 500 horas de funcionamento com água com alto risco de entupimento, sem que fossem submetidas a qualquer tipo de procedimento de limpeza, no esquema funcionando duas horas descansando uma hora até que o total de horas funcionando totalizasse 500 horas.

\subsection{Características da mangueira gotejadora e do emissores}

O experimento foi conduzido utilizando uma mangueira gotejadora de polietileno, com emissor coextrusado do tipo pastilha não-autocompensante (Figura 2), do tipo Tiran da marca Netafin. 


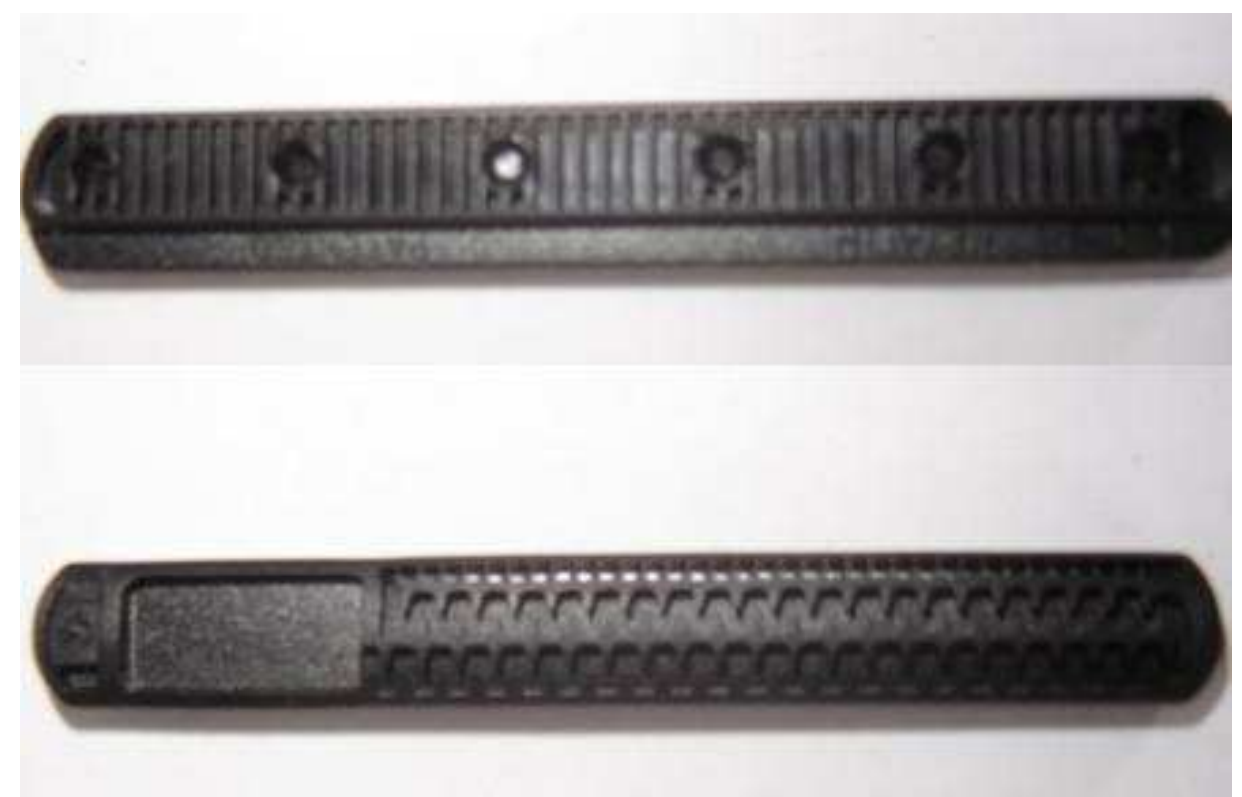

Figura 2. Perfis do emissor do tipo Tiran utilizado no experimento.

O diâmetro interno médio da mangueira gotejadora foi de $14,45 \mathrm{~mm}$, sendo medido por meio de um paquímetro digital (precisão de $0,01 \mathrm{~mm}$ ) com o auxílio de uma ferramenta cônica, conforme o apresentado pela norma ISO 9261:2004. Para determinação da espessura da parede da mangueira foram extraídas 10 amostras, ao acaso, e com o auxílio de um paquímetro digital com precisão de $0,01 \mathrm{~mm}$, determinou-se a espessura em quatro pontos eqüidistantes nas duas seções transversais ao comprimento da mangueira, calculando-se o valor médio.

$\mathrm{Na}$ Tabela 2 são apresentados os valores médios das características hidráulicas e geométricas do conjunto mangueira gotejadora e do emissor.

Para medir a área da seção do tubo emissor com o gotejador inserido, foi utilizada metodologia apresentada por Alves \& Porto (2002).

Foram feitos cortes transversais em cada tubo gotejador retirando-se ao acaso, 10 anéis com emissores inseridos para cada mangueira gotejadora, cujas larguras mediam aproximadamente $1,0 \mathrm{~cm}$. Em seguida, por meio de um scanner, digitalizou-se os conjuntos de perfis e com auxílio do Software Autocad, determinou-se as áreas das seções.

Tabela 2. Características hidráulicas e geométricas da mangueira e do emissor utilizada no experimento.

\begin{tabular}{lccc}
\hline Parâmetros & Média & $\begin{array}{c}\text { Desvio } \\
\text { Padrão }\end{array}$ & $\begin{array}{c}\text { Coeficiente } \\
\text { de variação } \\
(\%)\end{array}$ \\
\hline Diâmetro interno, $\mathrm{D}_{\mathrm{i}}(\mathrm{mm})$ & 14,45 & 0,12 & 0,83 \\
Diâmetro da seção com gotejador, $\mathrm{D}_{\mathrm{g}}(\mathrm{mm})$ & 13,29 & 0,23 & 1,73 \\
Espessura da parede $(\mathrm{mm})$ & 0,82 & 0,03 & 3,65 \\
Vazão média do emissor $\left(\mathrm{L} \cdot \mathrm{h}^{-1}\right)$ & 2,05 & 0,08 & 3,90 \\
Área da seção interna do tubo, $\mathrm{A}_{\mathrm{i}}\left(\mathrm{mm}^{2}\right)$ & 164,00 & 2,76 & 1,68 \\
Área da sec. interna do tubo com emissor, $\mathrm{A}_{\mathrm{g}}\left(\mathrm{mm}^{2}\right)$ & 139,54 & 1,60 & 1,15 \\
\hline
\end{tabular}

\subsection{Cálculo da perda de carga teórica}


Para o cálculo da perda de carga ao longo do comprimento da mangueira foi utilizada a equação de Darcy-Weisbach reescrita por Bagarello et al. (1995) (Equação 1). O valores de perda de carga ao longo do comprimento foram utilizados também na obtenção da perda de carga localizada subtraindo os valores de perda de carga obtidos em ensaio pelos valores obtidos pela Equação 1 .

No cálculo da perda de carga localizada na inserção do emissor, foi utilizada a equação geral da perda de carga localizada (Equação 2), com um coeficiente de carga cinética, $\alpha$, determinado pela Equação 3 apresentada por Provenzano \& Pumo em 2005.

Os resultados foram analisados estatisticamente, aplicando-se o teste $\mathrm{F}$ de acordo com seus respectivos esquemas de variância. Quando constatada a significância, as médias foram testadas pelo teste de Tukey a $\mathrm{P}<0,05$.

\section{RESULTADOS E DISCUSSÕES}

A Figura 3 demonstra a perda de carga observada em cada um dos pontos de medição, bem como suas componentes, a perda de carga ao longo do trecho e a perda de carga localizada devido à inserção do emissor.

Pode-se verificar que a perda de carga localizada é parte significativa do total, sendo em média $23,00 \%$ do valor da perda de carga total para esse modelo de mangueira gotejadora, não sendo possível desprezar tais perdas no dimensionamento do sistema de irrigação.

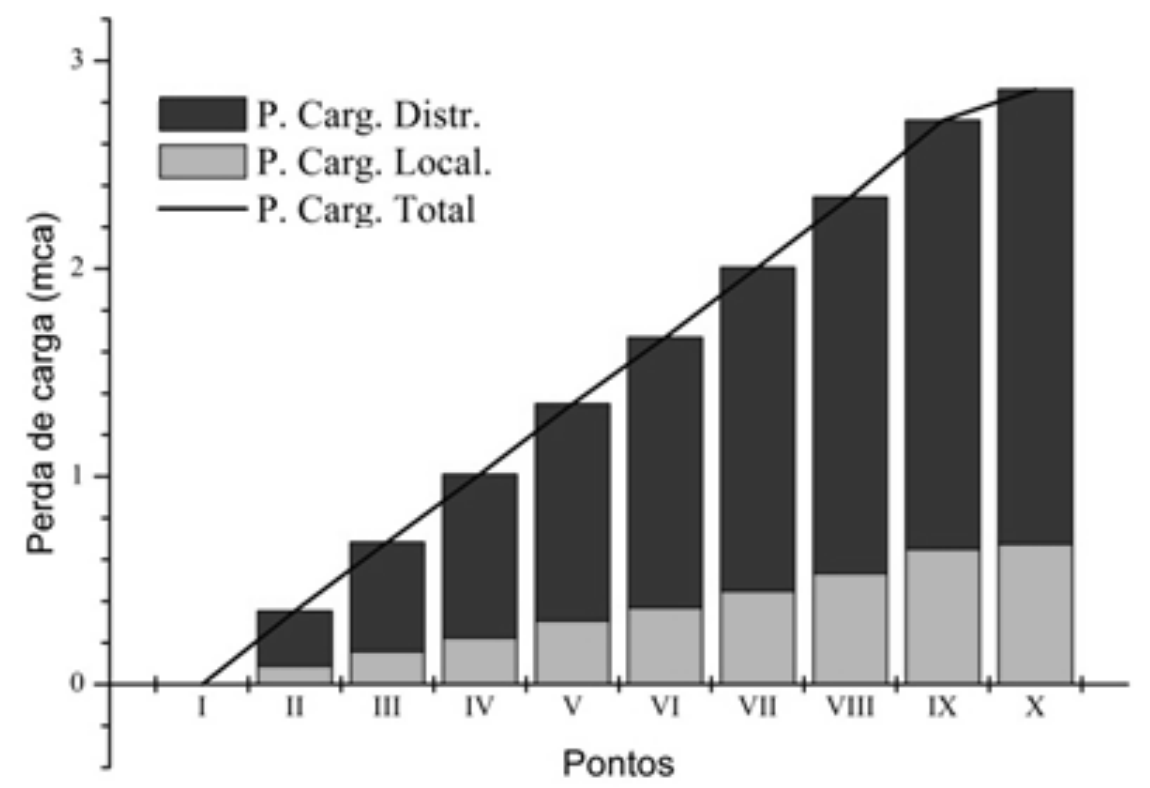

*P.Carg. Distr. - Perda de carga distribuída ao longo da linha; P.Carg.Local. - Perda de carga localizada ao longo da linha; P. Carg. Total - Soma da perda de carga distribuída e perda de carga localizada.

Figura 3. Gráfico do total de perda de carga e sua composição em perdas localizadas e distribuídas nos 9,2m de mangueira gotejadora ensaiados.

A Tabela 3 apresenta a comparação dos valores médios, através do teste de Tukey, da perda de carga localizada, gerada por cada um dos emissores, obtidos em ensaio e os obtidos através das equações. 
Verificou-se que a equação apresentou valores estatisticamente semelhantes à maioria dos valores encontrados nos ensaios para cada gotejador, segundo o teste de Tukey a $5 \%$ de significância. Portanto, para esse modelo de emissor, a equação apresenta bons resultados. Analisando-se as médias verifica-se que o valor obtido pelas equações para a perda de carga localizada foi de $0,0397 \mathrm{~m}, 6,4 \%$ maior que os $0,0373 \mathrm{~m}$ obtidos em ensaio.

Tabela 3. Valores de perda de carga localizada $(\mathrm{m})$ na inserção dos gotejadores.

\begin{tabular}{|c|c|c|}
\hline Emissores & $\begin{array}{l}\text { Perda de carga localizada } \\
\text { obtida em ensaio }(\mathrm{m})\end{array}$ & $\begin{array}{l}\text { Perda de carga localizada } \\
\text { obtida equação }(\mathrm{m})\end{array}$ \\
\hline 1 & $0,0295 \mathrm{~B}$ & $0,0406 \mathrm{~A}$ \\
\hline 2 & $0,0295 \mathrm{~B}$ & $0,0402 \mathrm{~A}$ \\
\hline 3 & $0,0295 \mathrm{~B}$ & $0,0402 \mathrm{~A}$ \\
\hline 4 & $0,0351 \mathrm{~A}$ & $0,0401 \mathrm{~A}$ \\
\hline 5 & $0,0351 \mathrm{~A}$ & $0,0402 \mathrm{~A}$ \\
\hline 6 & $0,0327 \mathrm{~B}$ & $0,0402 \mathrm{~A}$ \\
\hline 7 & $0,0327 \mathrm{~B}$ & $0,0401 \mathrm{~A}$ \\
\hline 8 & $0,0407 \mathrm{~A}$ & $0,0401 \mathrm{~A}$ \\
\hline 9 & $0,0407 \mathrm{~A}$ & $0,0400 \mathrm{~A}$ \\
\hline 10 & $0,0312 \mathrm{~A}$ & $0,0395 \mathrm{~A}$ \\
\hline 11 & $0,0312 \mathrm{~A}$ & $0,0394 \mathrm{~A}$ \\
\hline 12 & $0,0400 \mathrm{~A}$ & $0,0394 \mathrm{~A}$ \\
\hline 13 & $0,0400 \mathrm{~A}$ & $0,0393 \mathrm{~A}$ \\
\hline 14 & $0,0425 \mathrm{~A}$ & $0,0393 \mathrm{~A}$ \\
\hline 15 & $0,0425 \mathrm{~A}$ & $0,0392 \mathrm{~A}$ \\
\hline 16 & $0,0586 \mathrm{~A}$ & $0,0392 \mathrm{~B}$ \\
\hline 17 & $0,0586 \mathrm{~A}$ & $0,0391 \mathrm{~B}$ \\
\hline 18 & $0,0228 \mathrm{~A}$ & $0,0397 \mathrm{~A}$ \\
\hline Média & 0,0373 & 0,0397 \\
\hline
\end{tabular}

* Valores em uma mesma linha, seguidos de mesma letra são considerados estatisticamente semelhantes a 5\% de significância.

A Tabela 4 apresenta as médias da perda de carga total na linha ensaiada para o conjunto mangueira e emissores, quando novos e após 500 horas de funcionamento com uma água com alto risco de entupimento e a sua variação para cada um dos pontos de medição.

Verificou-se que o uso com a água rica em ferro ocasionou um aumento na perda de carga total da linha lateral, chegando a ter variação maior que $20 \%$, tal variação pode 
comprometer o funcionamento do sistema, ocasionando uma redução na uniformidade de aplicação.

Tabela 4. Valores da perda de carga total para mangueira gotejadora e emissores novos, após 500 horas de uso e sua variação

\begin{tabular}{llll}
\hline Pontos de Medição & P.C. - Novos(m) & P.C.- Usados(m) & Variação(\%) \\
\hline I & 0 & 0 & 0 \\
II & 0,3523 & 0,3476 & 1,3 \\
III & 0,6845 & 0,7603 & 11,1 \\
IV & 1,0101 & 1,1262 & 11,50 \\
V & 1,3503 & 1,5911 & 17,83 \\
VI & 1,6697 & 2,0119 & 20,50 \\
VII & 2,0053 & 2,4353 & 21,44 \\
VIII & 2,3443 & 2,8682 & 22,35 \\
IX & 2,7144 & 3,2620 & 20,17 \\
X & 2,8626 & 3,5089 & 22,60 \\
\hline
\end{tabular}

* P.C. -Novos - Perda de carga total da mangueira gotejadora com emissores novos; P.C. -Usados - Perda de carga total da mangueira gotejadora com emissores após 500 horas de uso com água rica em ferro;

A Figura 4 ilustra a variação da perda de carga total, apresentada na Tabela 4 e a variação da perda de carga localizada nos pontos de medição.

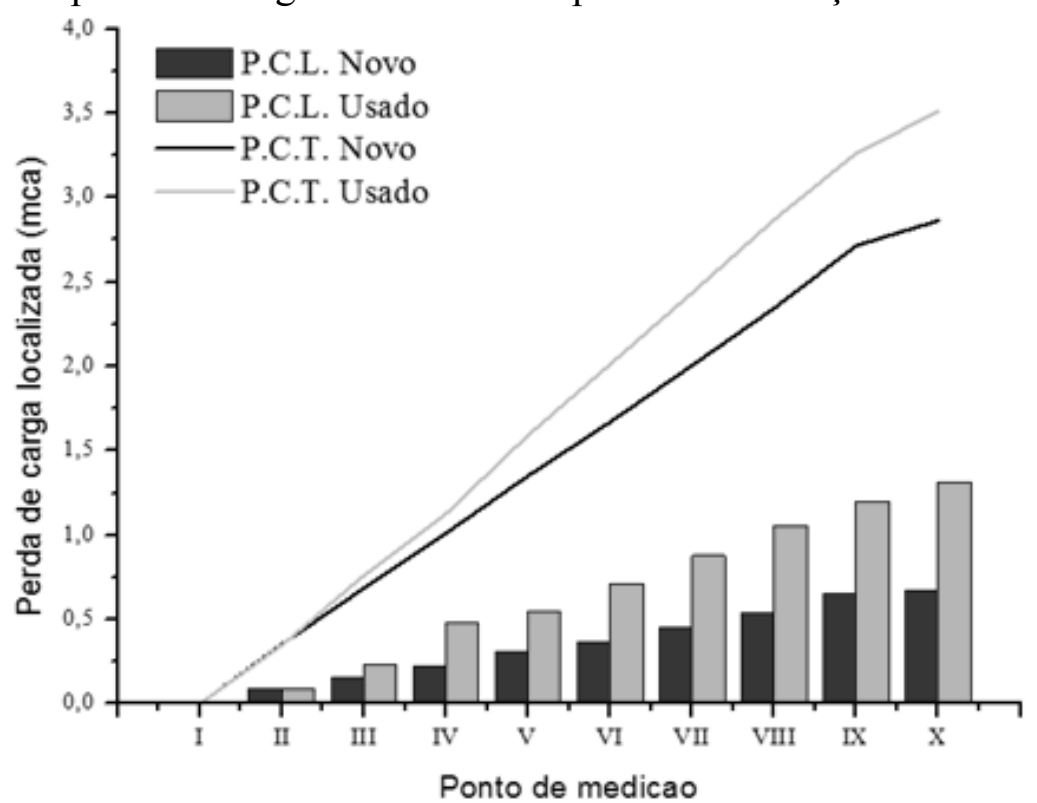

*P.C.L. Novo - Perda de carga localizada para emissores novos; P.C.L. Usado - Perda de carga para emissores após 500 horas de uso com água rica em ferro; P.C.T. Novo - Perda de carga total na linha com emissores novos; P.C.T. Usado - Perda de carga total na linha para emissores após 500 horas de uso com água rica em ferro. 
Figura 4. Comparação entre valores de perda de carga total e localizada para mangueira gotejadora nova e com 500 horas de uso.

Os valores da perda de carga localizada para o conjunto mangueira e emissores novos e usados estão apresentados na Tabela 5.

Tabela 5. Comparação entre os valores médios de perda de carga localizada, obtidos em ensaio, para gotejadores novos e após 500 horas de uso.

\begin{tabular}{lll}
\hline Pontos de Medição & P.C.L. - Novos(m) & P.C.L. - Usados(m) \\
\hline I & 0 & 0 \\
II & $0,0887 \mathrm{~A}$ & $0,0843 \mathrm{~A}$ \\
III & $0,1590 \mathrm{~B}$ & $0,2346 \mathrm{~A}$ \\
IV & $0,2244 \mathrm{~B}$ & $0,4760 \mathrm{~A}$ \\
V & $0,3060 \mathrm{~B}$ & $0,5464 \mathrm{~A}$ \\
VI & $0,3684 \mathrm{~B}$ & $0,7095 \mathrm{~A}$ \\
VII & $0,4486 \mathrm{~B}$ & $0,8769 \mathrm{~A}$ \\
VIII & $0,5337 \mathrm{~B}$ & $1,0548 \mathrm{~A}$ \\
IX & $0,6511 \mathrm{~B}$ & $1,1953 \mathrm{~A}$ \\
X & $0,6739 \mathrm{~B}$ & $1,3152 \mathrm{~A}$ \\
\hline lores em uma mesma linha, seguidos de mesma letra são considerados \\
ticamente semelhantes a 5\% de significância.
\end{tabular}

De acordo com os valores apresentados na Tabela 5 verificou-se que os valores de perda de carga localizada para cada ponto de medição variaram significativamente, de acordo com o teste de Tukey, o que indica que o conjunto mangueira gotejadora e emissores sofreram influencia da água utilizada.

\section{CONCLUSÕES}

Com base nos resultados obtidos durante o experimento conclui-se que:

- A perda de carga localizada representou $23 \%$ do valor da perda de carga total para esse modelo de mangueira gotejadora, o que ocasiona em um erro considerável ao desprezá-la no dimensionamento do sistema.

- A equação apresentada na literatura estimou de forma satisfatória a perda de carga localizada para o modelo de emissor utilizado no experimento, podendo assim, ser utilizada para se estimar a perda de carga localizada.

- A utilização da água com nível elevado de ferro ocasionou um aumento na perda de carga do sistema de aproximadamente $20 \%$ após 500 horas, refletindo num aumento significativo da perda de carga localizada, o que indica que esse conjunto, mangueira gotejadora e emissores, é suscetível a influencia do ferro no sistema. 


\section{REFERÊNCIAS}

ALVES, P. R. V.; PORTO, R. M. Coeficiente geométrico para estimativa da perda de carga localizada em linhas laterais de irrigação por gotejamento. Engenharia agrícola, Jaboticabal, v. 22, n. 1, p.51-59, jan. 2002.

BAGARELlO, V.; FERRO, V.; PROVENZANO, G.; PUMO, D. Experimental study on flow-resistance law for small-diameter plastic pipes. Journal of Irrigation and Drainage Engineering, New York, v. 121, n. 5, p. 313-316, Sep./Oct. 1995.

BERNARDO, S. ; SOARES, A. A.; MANTOVANI, E. C. Manual de irrigação. 8. ed. Viçosa, MG: UFV, 2006. 625p.

JUANA, L.; RODRÍGUEZ-SINOBÁS, L., LOSADA, A. Determining minor head loses in drip irrigation laterals. I: Methodology. Journal of Irrigation and Drainage Engineering, New York, v. 128, n. 6, p. 376-384, Nov./Dec. 2002.

PROVENZANO, G.; PUMO, D.; DIO, P. DI. Simplified procedure to evaluate head losses in Drip Irrigation Laterals. Journal of Irrigation and Drainage Engineering, New York, v. 131, n. 6, p.525-532, Nov./Dec. 2005.

WU, I.-P. An assessment of hydraulic design of micro-irrigation systems. Agricultural Water Management, New York, v. 32. n. 3, p. 275-284, April, 1997. 